\title{
DOMINATING VERTEX COVERS: THE VERTEX-EDGE DOMINATION PROBLEM
}

\author{
William F. KLOSTERMEYER \\ School of Computing \\ University of North Florida \\ Jacksonville, FL 32224 USA \\ e-mail: wkloster@unf.edu \\ Margaret-Ellen Messinger \\ Department of Mathematics and Computer Science \\ Mount Allison University, Sackville, NB, Canada \\ e-mail: mmessinger@mta.ca \\ AND \\ ANDERS YeO \\ Department of Mathematics and Computer Science \\ University of Southern Denmark \\ Odense, Denmark \\ and Department of Mathematics \\ University of Johannesburg \\ Auckland Park, 2006 South Africa \\ e-mail: yeo@imada.sdu.dk
}

\begin{abstract}
The vertex-edge domination number of a graph, $\gamma_{v e}(G)$, is defined to be the cardinality of a smallest set $D$ such that there exists a vertex cover $C$ of $G$ such that each vertex in $C$ is dominated by a vertex in $D$. This is motivated by the problem of determining how many guards are needed in a graph so that a searchlight can be shone down each edge by a guard either incident to that edge or at most distance one from a vertex incident to the edge. Our main result is that for any cubic graph $G$ with $n$ vertices, $\gamma_{v e}(G) \leq 9 n / 26$. We also show that it is $N P$-hard to decide if $\gamma_{v e}(G)=\gamma(G)$ for bipartite graph $G$.
\end{abstract}

Keywords: cubic graph, dominating set, vertex cover, vertex-edge dominating set.

2010 Mathematics Subject Classification: 05C69. 


\section{REFERENCES}

[1] R. Boutrig, M. Chellali, T.W. Haynes and S.T. Hedetniemi, Vertex-edge domination in graphs, Aequationes Math. 90 (2016) 355-366.

doi:10.1007/s00010-015-0354-2

[2] D. Dereniowski, H. Ono, I. Suzuki, Ł. Wrona, M. Yamashita and P. Żylinski, The searchlight problem for road networks, Theoret. Comput. Sci. 591 (2015) 28-59. doi:10.1016/j.tcs.2015.04.026

[3] M.A. Henning and A. Yeo, Transversals in 4-uniform hypergraphs, Electron. J. Combin. 23 (2016) \#P3.50.

[4] W.F. Klostermeyer and C.M. Mynhardt, Edge protection in graphs, Australas. J. Combin. 45 (2009) 235-250.

[5] W.F. Klostermeyer and C.M. Mynhardt, Protecting a graph with mobile guards, Appl. Anal. Discrete Math. 10 (2016) 1-29. doi:10.2298/AADM151109021K

[6] A.V. Kostochka and C. Stocker, A new bound on the domination number of connected cubic graphs, Sib. Ėlektron. Mat. Izv. 6 (2009) 465-504.

[7] B. Krishnakumari, Y.B. Venkatakrishnan and M. Krzywkowski, Bounds on the vertex-edge domination number of a tree, C.R. Math. 352 (2014) 363-366. doi:10.1016/j.crma.2014.03.017

[8] J.R. Lewis, Vertex-Edge and Edge-Vertex Domination in Graphs, Ph.D. Thesis (Clemson University, Clemson, 2007).

[9] J.R. Lewis, S.T. Hedetniemi, T.W. Haynes and G.H. Fricke, Vertex-edge domination, Util. Math. 81 (2010) 193-213.

[10] J.W. Peters, Theoretical and Algorithmic Results on Domination and Connectivity, Ph.D. Thesis (Clemson University, Clemson, 1986).

[11] Y.B. Venkatakrishnan, C. Natarajan and G. Sathiamoorthy, Vertex-edge and connected domination numbers of a tree, Int. J. Pure Appl. Math. 119 (2018) 103-111.

[12] W.C.K. Yen and C.Y. Tang, An optimal algorithm for solving the searchlight guarding problem on weight two-terminal series-parallel graphs, Acta Inform. 36 (1999) 143-172.

doi:10.1007/s002360050156

Received 4 January 2018

Revised 25 August 2018

Accepted 25 August 2018 